\title{
Numerical comparison of reduced order models for non-linear vibrations of damped plates
}

\author{
F. Boumediene ${ }^{\mathrm{a}, \mathrm{b}}$, L. Duigou ${ }^{\mathrm{a}}$, A. Miloudi ${ }^{\mathrm{b}}$ and J.M. Cadou ${ }^{\mathrm{a} *}$ \\ ${ }^{a}$ Laboratoire d'Ingénierie des Matériaux de Bretagne, Université Européenne de Bretagne, Université de \\ Bretagne Sud, Rue de Saint Maudé, BP 92116, 56321 Lorient cedex, France; ${ }^{b}$ Laboratoire de \\ Mécanique Avancée, Faculté de Génie Mécanique \& Génie des Procédés, USTHB, BP 32, El Alia, \\ 16111 Bab Ezzouar, Algiers, Algeria
}

\begin{abstract}
This work deals with the computation of the non-linear solutions of the vibration of damped plates by coupling a harmonic balance method and the asymptotic numerical method. These computations can lead to lengthy central processing unit (CPU) times if the solution sought contains an important number of harmonics. In this study, we propose two reduced order models which can be applied to solve this type of problem. Both reduced methods are based on a first computation carried out with a small number of harmonics (here two). Numerical examples of plate vibration show that these algorithms help save a great deal of computational time and can be applied to problems involving numerous harmonics.
\end{abstract}

Ce travail porte sur le calcul des solutions non linéaires de vibration des plaques minces amorties par le couplage de la méthode d'Equilibrage Harmonique et la Méthode Asymptotique Numérique (MAN). Ces calculs peuvent conduire à un temps significatif si la solution recherchée contient un nombre important d'harmoniques. Dans cette étude, nous proposons deux modèles pour réduire le temps de calcul. Les deux méthodes sont basées sur un premier calcul réalisé avec un petit nombre d'harmoniques (ici deux). Des exemples numériques de plaques montrent que ces algorithmes aident à économiser beaucoup de temps et peuvent être appliqués à des problèmes impliquant de nombreuses harmoniques.

Keywords: non-linear vibration; damping; asymptotic numerical method; harmonic balance method; plates; reduced order model; proper orthogonal decomposition

Mots-clés: vibrations non linéaires; amortissement; méthode asymptotique numérique; méthode d'équilibrage harmonique; plaque; modèle d'ordre réduit; méthode orthogonale aux valeurs propres

\section{Introduction}

Today, thin plates are widely used in industrial applications including in aeronautics or the automobile industry. This type of structure generates the drawback of vibrating with large amplitudes. These large displacements are mainly due to their small thickness leading to geometrical non-linearities. The simulation of these problems thus requires efficient numerical methods. Classical methods consist of coupling temporal discretisation with an iterative method. The latter can be, for example, the Newton method. An alternative to the previous

*Corresponding author. Email: jean-marc.cadou@univ-ubs.fr 
methods is the harmonic balance method (HBM) (LaBryer \& Attar, 2010; Mickens, 2010) which is well adapted to time-periodic problems. This method enables the transformation of the initial time-dependent problem into a static non-linear one. This non-linear problem is then solved by using, for example, the Newton algorithm or the asymptotic numerical method (ANM) (Cochelin, Damil, \& Potier-Ferry, 1994). The latter includes the advantage of providing analytic solutions with smaller computational times than the ones required for a Newton scheme (Abdoun, Azrar, Daya, \& Potier-Ferry, 2009).

The main drawback of the HBM is that the size of the problem can be considerably increased if the number of harmonics required in the study is important. Consequently, the computational times increase dramatically. In this work, specific numerical methods are proposed to deal with the substantial size of the problem leading to a considerable computational time. Over the last two decades, various works have focused on reducing the computation time required to study such problems. Specific linear solvers which are well adapted to ANM can be chosen. One can quote, for example, the works of Médale and Cochelin (2009) or Cadou and PotierFerry (2010). Another way to decrease computational time is to use reduced order models.

In a recent work (Boumediene, Duigou, Boutyour, Miloudi, \& Cadou, 2011), we have developed a reduced method to compute the dynamical response of thin damped plate. This method is based on the projection of the unknown vector onto a matrix built from a previous computation of the same problem. Hence, the first step of calculation is done on the full size problem; the following computations (or following steps) are carried out on a reduced size problem obtained with the help of the previous computed quantities. The drawback of such an approach is that the reduction technique requires a first computational step, on the full size problem, which can be difficult to do if the number of harmonics is substantial or if the number of unknowns is great.

In this paper, we suggest two reduction procedures which are not based on computations on the full size problem. The first one is quite identical to the method proposed in Boumediene et al. (2011), but the projection basis is defined with a calculation carried out with a number of harmonics lower than the initial problem. For example, the full size problem requires six harmonics but the projection basis is built with a computation done with two harmonics only. The second method studied in this work is among the most useful reduction techniques in the field of fluid or solid mechanics: the proper orthogonal decomposition (POD) (Holmes, Lumley, \& Berkooz, 1996; Wriggers, 2008; Yvonnet \& He, 2007). This method requires a first computation (or a prior knowledge of the solution) to construct the projection basis. In order to avoid long computational times, this first computation is carried out, on a problem including a small number of harmonics (two), as for the first reduction technique. Thus, from this first computation a snapshot matrix is built by considering either the time or the circular frequency as parameter. Therefore in this study, the POD is coupled with the ANM to provide an efficient numerical tool.

\section{Problem formulation}

Let us consider a thin rectangular plate with a coordinate system $(O, x, y, z)$. The plate is submitted to multiharmonic time-dependent excitation in the $z$ direction of the form:

$$
\mathbf{P}(x, y, z, t)=\sum_{\mathrm{j}=0}^{H-1}\left(\mathbf{P}^{\mathrm{jc}}(x, y, z) \cos j \omega t+\mathbf{P}^{\mathrm{j} s}(x, y, z) \sin j \omega t\right)
$$

where $H$ is the number of harmonics, $t$ is the time, $\omega$ is the circular frequency, symbols $\mathrm{c}$ and $\mathrm{s}$ denote, respectively, the cosine and sine factors and $j$ is the harmonic number with. 
The displacement components of the plate's middle surface are denoted by vector $\mathbf{U}$. As the excitation is multiharmonic, the displacement response of the plate is supposed to be also multiharmonic and it can be written in the following form:

$$
\mathbf{U}(x, y, z, t)=\sum_{\mathrm{j}=0}^{H-1}\left(\mathbf{U}^{\mathrm{jc}}(x, y, z) \cos j \omega t+\mathbf{U}^{\mathrm{js}}(x, y, z) \sin j \omega t\right)
$$

With application of the von Karman theory, the Harmonic Balance Method (HBM) and the discretisation of the plate into ND degrees of freedom (dof) by the finite element method (FEM), the governing equation is cubic with respect to unknown parameters $(\omega, \mathbf{U})$ as follows (Boumediene, Miloudi, Cadou, Duigou, \& Boutyour, 2009):

$$
\mathbf{K U}^{3}+\omega \mathbf{C} \mathbf{U}-\omega^{2} \mathbf{M} \mathbf{U}=\mathbf{P}
$$

where $\mathbf{M}$ is the mass matrix, $\mathbf{K}$ is the stiffness matrix, $\mathbf{C}$ is the viscous damping matrix (Rayleigh type $\mathbf{C}=\alpha \mathbf{M}+\beta \mathbf{K}_{\mathbf{l}}, \mathbf{K}_{\mathbf{l}}$ is the elastic stiffness matrix) and $\mathbf{P}$ and $\mathbf{U}$ are written as follows:

$$
\begin{aligned}
\mathbf{P} & =\left[\begin{array}{llllll}
\mathbf{P}^{0} & \mathbf{P}^{1 \mathrm{c}} & \mathbf{P}^{1 \mathrm{~s}} & \cdots & \mathbf{P}^{(H-1) \mathrm{c}} & \mathbf{P}^{(H-1) \mathrm{s}}
\end{array}\right] \\
\mathbf{U} & =\left[\begin{array}{llllll}
\mathbf{U}^{0} & \mathbf{U}^{1 \mathrm{c}} & \mathbf{U}^{1 \mathrm{~s}} & \cdots & \mathbf{U}^{(H-1) \mathrm{c}} & \mathbf{U}^{(H-1) \mathrm{s}}
\end{array}\right]
\end{aligned}
$$

Then, the problem has ND $\times(2 H-1)$ dof, where ND is the dimension of $\mathbf{U}^{\mathbf{j} \mathbf{c}}$ and $\mathbf{U}^{\mathbf{j s}}$ $(\mathbf{j}=0, H-1)$.

The problem is to solve system (3) in which the unknowns are the displacement vector and the frequency. In order to simplify the use of the ANM, some rearrangements can be done to rewrite this equation in quadratic form (Cochelin \& Vergez, 2009; Potier-Ferry et al., 1997). To do so, new unknowns should be added to Equation (3): the stress vector $\mathbf{N}$ of dimension $\mathrm{ND} \times(2 H-1)$ and the scalar $\Omega=\omega^{2}$. The displacement, the frequency and the additional unknowns constitute one unknown vector $\mathbf{X}^{T}=\left(\begin{array}{llll}\omega & \Omega & \mathbf{U}^{T} & \mathbf{N}^{T}\end{array}\right)$ of dimension $\mathrm{NT}=2+2 \times \mathrm{ND} \times(2 \mathrm{H}-1)$ the non-linear governing equation is written then in the following form (Boumediene et al., 2011):

$$
\mathbf{R}(\mathbf{X})=0
$$

In order to solve Equation (6), the ANM is used, which is a continuation technique where the non-linear problem is transformed into a sequence of linear ones. This method has been successfully used in different fields, such as in fluid mechanics (Cadou, Cochelin, Damil, \& Potier-Ferry, 2001) or recently to solve the fracture problem (Daridon, Wattrisse, Chrysochoos, \& Potier-Ferry, 2011).

\section{Asymptotic numerical method}

Within asymptotic-numerical techniques, the unknown $\mathbf{X}$ is sought in the form of truncated power series with respect to a path parameter ' $a$ ': 


$$
\mathbf{X}(a)=\mathbf{X}_{0}+\sum_{p=1}^{n} a^{p} \mathbf{X}_{p}
$$

$\mathbf{X}_{0}$ is a regular solution of the non-linear problem (6) and $\mathbf{X}_{p}$ is the unknown, at each order $p(p=1, n)$ to be computed. $n$ is the truncation order.

The series (7) are inserted in Equations (6). Then, from the identification of the like powers of ' $a$ ', a set of recurrent linear problems is obtained at each order $p$ as follows:

$$
\mathbf{K}_{\mathbf{t} 0} \mathbf{X}_{p}=\mathbf{F}_{p}^{\mathbf{n l}}
$$

where $\mathbf{X}_{p}$ is the unknown vector at order $p, \mathbf{F}_{p}^{\mathbf{n l}}$ is the second member vector at order $p$ computed in function to known parameters $\mathbf{X}_{i}$ given in precedent orders $(i=1, p-1)$ and $\mathbf{K}_{\mathbf{t} 0}$ denotes the Jacobian matrix at the initial point $\mathbf{X}_{0}$.

The governing system has one more unknowns than equations. Then, another equation must be added. To do so, the path parameter ' $a$ ' is identified as the projection of the unknown vector increment $\left(\mathbf{X}-\mathbf{X}_{0}\right)$, on the tangent vector $\mathbf{X}_{1}$ :

$$
a={ }^{T} \mathbf{X}_{1} \mathbf{Z}\left(\mathbf{X}-\mathbf{X}_{0}\right)
$$

where $\mathbf{Z}$ is a diagonal matrix, the components of which are equal to 1 or 0 to define the ANM parameterisation (Mottaqui, Braikat, \& Damil, 2010). In this study, a pseudo arc-length scheme is used. The implementation of Equation (7) in the latter (9) makes it possible to provide the additional equation at each order (Boumediene et al., 2009).

These asymptotic expansions have a limited range of validity which can be evaluated by using a simple criterion (Cochelin et al., 1994). The maximal value ' $\mathrm{a}_{\max }$ ' of the path parameter ' $a$ ' is defined using the inequality (10): the relative difference between the displacements at two consecutive orders must be smaller than a given parameter (choosing as the ANM tolerance parameter $\eta$ ), which leads to:

$$
\left\|\mathbf{U}_{n}-\mathbf{U}_{n-1}\right\| /\left\|\mathbf{U}_{n}-\mathbf{U}_{0}\right\| \leq \eta
$$

The maximum value of the path parameter, $a_{\max }$ is computed from the latter expression. Then, $a_{\max }$ is introduced in (7) to compute a new starting point $\mathbf{X}_{0}$. From this point, one can apply the perturbation method and compute a supplementary part of the non-linear solution branch. This defines the so-called continuation method and enables the computation, of the non-linear solution branch in a step-by-step method. Nevertheless, as it has been previously shown (Elhage-Hussein, Potier-Ferry, \& Damil, 2000), the best path algorithm is the one based on the Padé approximants: the polynomial representation is replaced by rational fractions named Padé approximants.

At this stage, the equation to be solved has a high number of dof. In order to reduce them, as well as the computation time, the reduced order models are introduced in the discrete form (8). This procedure is described in the following section.

\section{Reduction model}

Reduction model is applied at each order on the linear problem (8) obtained after using ANM and FEM. In order to reduce the number of dof, the unknown vectors of dimension NT are projected onto a low-dimensional subspace as follows: 


$$
\mathbf{X}_{p}=\boldsymbol{\Phi} \mathbf{x}_{p}
$$

where $\mathbf{x}_{p}$ is the reduced unknown vector of dimension $n t=2+2 \times \mathrm{nd} \times(2 H-1)(\mathrm{nt}<\mathrm{NT})$ and $\boldsymbol{\Phi}$ is the projection basis (projection matrix) of dimension NT $\times \mathrm{nt}$.

$\mathbf{X}_{\mathrm{p}}$ is substituted by expression (11), in Equation (8). Multiplying the left of each side by the transpose of the projection matrix $\boldsymbol{\Phi}$, Equation (8) becomes:

$$
\mathbf{k}_{\mathbf{t} \mathbf{0}} \mathbf{x}_{p}=\mathbf{f}_{p}^{\mathbf{n l}}
$$

where $\mathbf{k}_{\mathbf{t} \mathbf{0}}$ and $\mathbf{f}_{p}^{\mathrm{nl}}$, respectively, denote the reduced forms of $\mathbf{K}_{\mathbf{t 0}}$ and $\mathbf{F}_{p}^{\mathbf{n l}}$ given in Equation (8), where:

$$
\begin{gathered}
\mathbf{k}_{\mathbf{t} \mathbf{0}}=\boldsymbol{\Phi}^{T} \mathbf{K}_{\mathbf{t 0}} \boldsymbol{\Phi} \\
\mathbf{f}_{p}^{\mathrm{nl}}=\boldsymbol{\Phi}^{T} \mathbf{F}_{p}^{\mathrm{nl}}
\end{gathered}
$$

In order to avoid a lengthy computing time and great size operators to be stored, the projection method is applied to the elementary level before the assembly of the tangent matrix $\mathbf{K}_{\mathbf{t 0}}$ and the second member $\mathbf{F}_{p}^{\mathrm{nl}}$.

\subsection{Reduction method based on a non-linear calculation: non-linear basis}

The reduction model is built from the resolution of the dynamical problem. One ANM step is achieved without reduction. The vector calculated at each order $p$ is then used to construct the projection matrix. This basis is denoted non-linear basis (NLB), because these columns are the results of a non-linear computation. This makes it possible to introduce the non-linear effect in the projection basis to improve the solution quality. This reduction technique has already been studied in a previous work (Boumediene et al., 2011). The novelty here is that the first ANM step, giving the non-linear vectors for the projection, is made with two harmonics only, whatever the harmonic number is for the response study. This preliminary calculation is done with one harmonic excitation force defined as follows:

$$
\mathbf{P}=\mathbf{P}^{1 \mathbf{c}} \cos \omega t
$$

and by considering two harmonics only in the displacement vector. Then, the displacement and the force vectors are given, respectively, as follows:

$$
\begin{aligned}
& \mathbf{U}=\left[\begin{array}{lll}
\mathbf{U}^{0} & \mathbf{U}^{1 \mathrm{c}} & \mathbf{U}^{1 \mathrm{~s}}
\end{array}\right] \\
& \mathbf{P}=\left[\begin{array}{lll}
\mathbf{0} & \mathbf{P}^{1 \mathrm{c}} & \mathbf{0}
\end{array}\right]
\end{aligned}
$$

$\mathbf{U}$ and $\mathbf{P}$ have dimension $\mathrm{ND} \times(2 H-1)$ (here, $H=2)$.

Finally, this database is used to calculate the answer to a problem containing a harmonic number greater than 2 and to a multiharmonic excitation. The advantage of this approach is that it avoids the construction of large matrices and it reduces the required computational times for the basis definition which is the drawback of the proposed method in Boumediene 
et al. (2009). The displacement vector is computed then in a reduced form with dimension nd $\times(2 H-1)$ only. After that, the full size order ND $\times(2 H-1)$ displacement vector is built.

\subsection{Proper orthogonal decomposition}

The second way to define the projection operators in this study is based on the POD. This method has been initially built to analyse data from random processes by introducing new systems based on statistics. Lately, it has become a very effective method for multiple tasks of data analysis, compression and model reduction. Applications of this approach are found in several disciplines: analysis of random variables, image processing, signal analysis, data compression, process identification, chemical engineering, oceanography, etc. The POD is a very effective technique for analysing data in order to approximate a system of large dimension by another of much smaller dimension. This method is a linear process, enabling to determine a basis of the most probable representative orthogonal eigenmodes (Holmes et al., 1996; Wriggers, 2008). The POD is applied with success in different fields even in non-linear vibrations (Amabili, Sarkar, \& Paidoussis, 2003; Antoulas \& Sorensen, 2001; Goncalves, Silva, \& Del Prado, 2008; Lall, Krysl, \& Marsden, 2003). The POD can be applied on a discrete interval named snapshot matrix (for more details the reader can see references cited in this paragraph). This snapshot matrix is calculated in function to a parameter defining the process and generally referring to time, but it can also refer to the load. Here, the POD is applied on snapshot matrices using either the time or the excitation frequency as parameter.

To use the POD method, a first computation has to be made by ANM without reduction, in a large interval around the first mode to gather a lot of information on dynamical response. The basis is built for an excitation form given by (13) using two harmonics only. From this calculation, a snapshot matrix is built by changing either the excitation circular frequency or the time parameter by setting the circular frequency value. Bases built from frequency and time snapshot matrices are noted POD- $\omega$ and POD- $t$, respectively.

\section{Numerical discussion}

Consider a simply supported, isotropic and homogeneous rectangular plate. Its dimensions are: length $L=.6 \mathrm{~m}$, width $l=.3 \mathrm{~m}$ and thickness $h=.001 \mathrm{~m}$. The material is aluminium whose
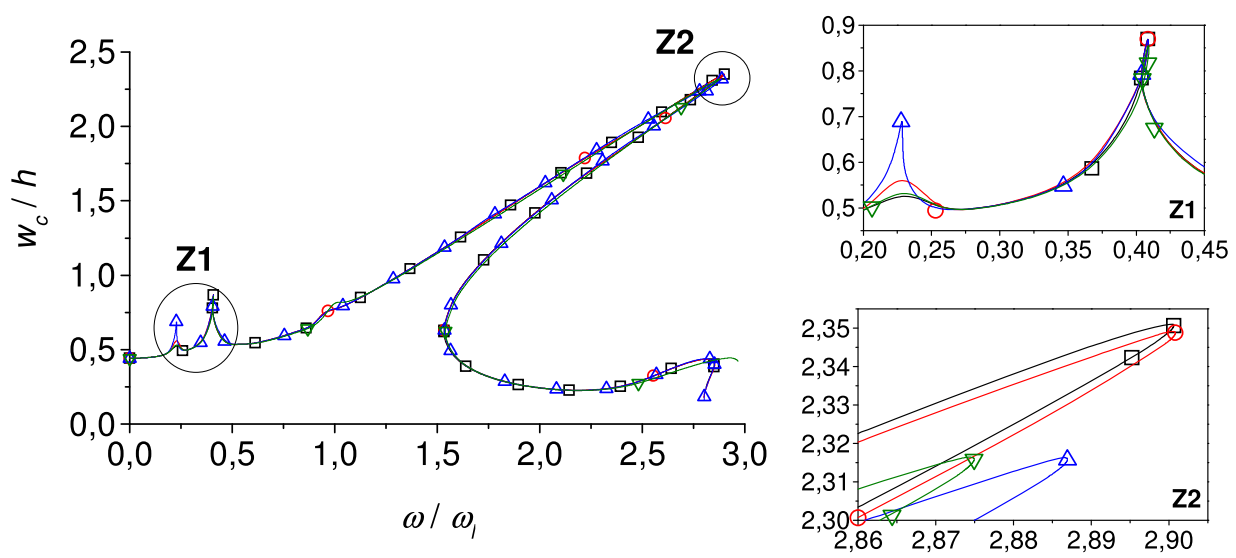

Figure 1. Plate dynamic response using ANM $(H=6, \mathrm{P}$ (Equation (11)) + NLB and POD (base: $H=2$, $P$ (Equation (11)). $\rightarrow$ ANM; $\multimap-\mathrm{ANM}+\mathrm{NLB}(\mathrm{nd}=15) ; \multimap-\mathrm{ANM}+\mathrm{POD}-\omega(\mathrm{nd}=8) ; \rightarrow$ ANM + POD- $t(\mathrm{nd}=8)$. 
characteristics are: Young's modulus $E=70,109 \mathrm{~Pa}$, density $\rho=2778 \mathrm{~kg} / \mathrm{m}^{3}$ and Poisson's ratio $v=.3$. The damping matrix is proportional to the elastic stiffness matrix $\left(\mathbf{K}_{\mathbf{l}}\right)$ with a coefficient $\beta=.0001\left(\mathbf{C}=\beta \cdot \mathbf{K}_{\mathbf{l}}\right)$. This plate is subjected to a harmonic exciting force perpendicular to the mean surface and uniformly distributed $P=40 \mathrm{~N} / \mathrm{m}^{2}$. The force vector has the form (13).

The plate is modelled using DKT triangular shell elements with three nodes and six dof per node $(u, v, w, \theta x, \theta y, \theta z)$ (Batoz \& Dhatt, 1992). For reasons of symmetry, only one quarter of the plate is modelled and discretised into 435 nodes (i.e. 2610 dof for one harmonic bloc). The plate first natural frequency $\omega_{1}$ is equal to $208.67 \mathrm{rad} / \mathrm{s}$. Depending on previous work (Boumediene et al., 2011), the following ANM parameters are used: tolerance $\eta=10^{-4}$ and the order of truncation of the asymptotic approximations $n=20$. These parameters are used in all calculations. For the decomposition of the tangent matrix, we used the classical Crout method.

In this section, the three procedures (NLB, POD- $\omega$ and POD- $t$ ) are compared and applied for resolution with higher harmonic number. These numerical tests make it possible to validate the proposed reduction procedures. For the case $H=6$ (Figure 1), response curves found by reduction models are compared to those obtained by ANM without reduction. The NLB matrix contains 15 vectors whereas the bases POD- $t$ and POD- $\omega$ contain eight vectors (number selected by an energy-related criterion). One notes in Figure 1 that all the curves coincide perfectly. Only a small difference is visible at the second symmetrical mode. Around this mode, POD- $\omega$ and NLB give good results. However, for large amplitudes, one notes that the NLB gives the best results.

Previous results demonstrate that bases constructed from computation with two harmonics can give good results for problems involving higher harmonic number $(H=6)$. They are used hereafter to compute the dynamical responses for multiharmonic excitation type in order to check the validity range of these bases. To do so, the plate is now submitted to multiharmonic excitation of the form:

$$
\begin{gathered}
\mathbf{P}(\mathbf{t})=\mathbf{P}^{0}+\mathbf{P}^{1 \mathrm{c}} \cos \omega t+\mathbf{P}^{1 \mathrm{~s}} \sin \omega t ; \quad \mathbf{P}^{0}=\mathbf{P}^{1 c}=\mathbf{P}^{1 s} \\
\mathbf{P}=\underbrace{\left(\begin{array}{llllll}
\mathbf{P}^{0} & \mathbf{P}^{1 \mathrm{c}} & \mathbf{P}^{1 \mathrm{~s}} & \mathbf{0} & \cdots & \mathbf{0}
\end{array}\right)}_{(2 H-1) \text { blocs }}
\end{gathered}
$$

In all the tests, the bases are the same as the ones used in the previous example. More precisely, the bases are built with an excitation load given in expression (13), which is not the one considered in the full size problem (15).

In Figure 2, the response curves obtained with the three reduction techniques are compared to the one computed with the classical ANM. These results correspond to the excitation given by expression (15) and by taking six harmonics $(H=6)$ into account. In these curves, one can see that the solution diverges before the resonance when the basis is constructed from snapshots on time (Figure 2). Contrariwise, bases issued from POD- $\omega$ with snapshots on frequency and NLB give very efficient solutions compared to those obtained with classical ANM.

Figure 3 shows the dynamical response by taking 14 harmonics in the response $(H=14$ in (15)). The reduction methods (POD- $\omega$ and NLB) perfectly follow the classical ANM solutions even when the harmonic number of the full size problem is large. Then, it does not seem necessary to reconstruct the basis for computations differing in the applied loads. 

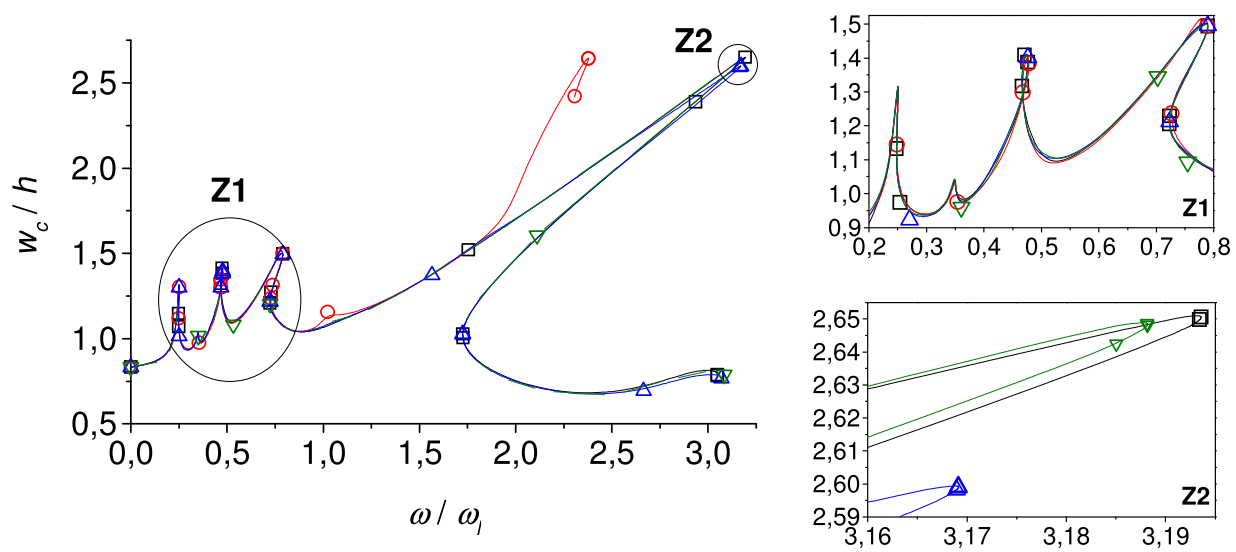

Figure 2. Plate dynamic response using ANM $(H=6, P$ (Equation (15)), NLB and POD (base: $H=2$, $P$ (Equation (13)). $\rightarrow$ ANM; $\multimap$ ANM + POD- $t(\mathrm{nd}=15) ; \multimap-\mathrm{ANM}+\mathrm{POD}-\omega(\mathrm{nd}=10) ; \rightarrow$ ANM + NLB $(\mathrm{nd}=10)$.
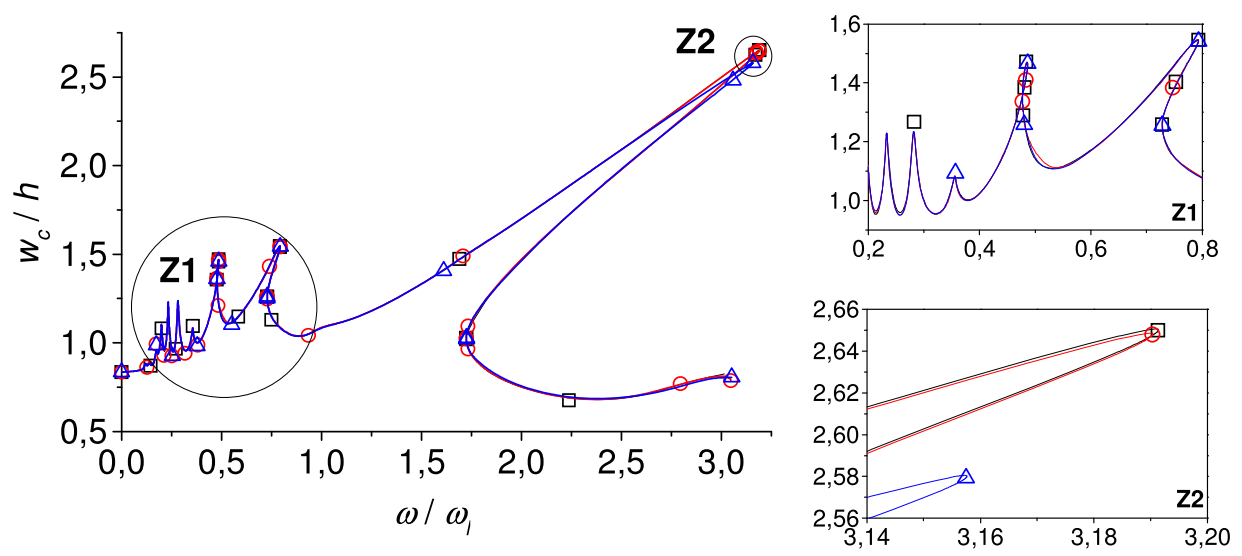

Figure 3. Plate dynamic response using ANM ( $H=14, P$ (Equation (15)), NLB and POD (base: $H=2$, $P($ Equation (13)). $\square-$ ANM; $\multimap-$ ANM + NLB (nd $=20) ; \multimap-$ ANM + POD- $\omega($ nd $=20)$.

The previous results validate two of the reduced order models proposed in this study: the NLB and the POD- $\omega$. The POD- $t$ can lead to divergence in the non-linear response curves when the considered number of harmonics is great. In the following section, a computational CPU time analysis is proposed to evaluate the benefit of using the reduced order models.

\subsection{Computational time}

This section is devoted to the comparison of the computational times needed by the classical ANM and the reduced order models proposed in this study. A plate subjected to a multiharmonic excitation vector is considered. Then, problems of dimension $2+2 \times \mathrm{nd} \times(2 H-1)$ are resolved instead of $2+2 \times \mathrm{ND} \times(2 H-1)$. In order to construct the projection basis, full order problems should be resolved. To do so, only one harmonic excitation force is taken into account and $H$ is taken equal to two. Then, the problem of dimension $(2+6 \times \mathrm{ND})$ is resolved to construct projection bases. 
Table 1. Computational time to get result around the first eigenmode $\omega \in\left[0,2 \omega_{l}\right]$. (2610 DOF).

\begin{tabular}{lllll}
\hline$H$ & & ANM & POD $-\omega(\mathrm{nd}=10)$ & NLB $(\mathrm{nd}=20)$ \\
\hline 2 & Basis constructing time & & $17 \times 50=850 \mathrm{~s}$ & $17 \mathrm{~s}$ \\
6 & One step time & $575 \mathrm{~s}$ & $50 \mathrm{~s}$ & $58 \mathrm{~s}$ \\
& CPU time (step number) & $75,325 \mathrm{~s} \cong 20 \mathrm{~h} 55^{\prime}$ & $6950 \mathrm{~s} \cong 1 \mathrm{~h} 56^{\prime}(122)$ & $7267 \mathrm{~s} \cong 2 \mathrm{~h}^{\prime}{ }^{\prime}(125)$ \\
& & $(131)$ & & \\
14 & One step time & $7647 \mathrm{~s} \cong 2 \mathrm{~h} 8^{\prime}$ & $315 \mathrm{~s}$ & $376 \mathrm{~s}$ \\
& CPU time (step number) & - & $41,485 \mathrm{~s} \cong 11 \mathrm{~h} 32^{\prime}$ & $50,401 \mathrm{~s} \cong 14 \mathrm{~h}(134)$ \\
& & $(129)$ & \\
\hline
\end{tabular}

The computational times for one step and the time needed to draw the curve around the first mode in the interval $\left[0,2 \omega_{1}\right]$ are shown in Table 1 for two harmonic numbers $H=6$ and $H=14$. This table also gives the computational times required by a classical ANM calculus.

The results in the Table 1 show that the two reduction methods give nearly the same gain. The POD- $\omega$ requires more computational times than the NLB to construct the basis. Nevertheless, the POD- $\omega$ makes it possible to select the dominant vectors and finally leads to a number of vectors in the basis which is two times lower than the NLB. This small number of vectors can then explain why both methods need approximately the same computational times to determine the whole response curve.

This time-saving is very significant. One can note that to find the solution around the first mode, the gain with six harmonics is around $91 \%$. For $H=14$, we do not do all the computation around the first mode with classical ANM because it can last up to over 11 days of calculation. Nevertheless, for one ANM step with $H=14$, the gain with a reduction method is around $96 \%$.

\section{Conclusion}

In this work, we propose two reduced order models to compute the non-linear response curve of damped plates. A first reduction technique, denoted by NLB, is an improvement of a previous published method (Boumediene et al., 2011) and the second one is based on a POD- $\omega$ analysis. Both methods are issued from a first computation carried out on a problem involving a small number of harmonics $(H=2$ in all our numerical tests). For the NLB technique, a single step of ANM is carried out and makes it possible to define an efficient basis. For the POD- $\omega$, several steps on a chosen range of the angular frequency are carried out. From these computations, two snapshot matrices are built: one with time as parameter and the second one with the circular frequency, respectively, denoted by POD- $t$ and POD- $\omega$. From the numerical tests implemented in this study, it appears that NLB and the POD- $\omega$ give similar results. Both methods are able to find the response curves, whereas, the POD- $t$ technique is not as efficient as the two previous ones. The accuracy of the solution obtained with the POD- $\omega$ and NLB is relatively good compared to those of the full size model and this with a gain of computing times around $90 \%$.

Nevertheless, NLB requires less computing time to construct the basis and seems to give results which are a little more accurate than those obtained with the POD- $\omega$. Moreover, the NLB technique is now applied to study the stability of fluid flows, more specifically for the determination of Hopf bifurcation points (Brezillon, Girault, \& Cadou, 2010) where the computing times considerably increase with the required number of unknowns. 


\section{References}

Abdoun, F., Azrar, L., Daya, E.M., \& Potier-Ferry, M. (2009). Forced harmonic response of viscoelastic structures by an asymptotic numerical method. Computers \& Structures, 87, 91-100.

Amabili, M., Sarkar, A., \& Pardoussis, M.P. (2003). Reduced-order models for nonlinear vibrations of cylindrical shells via the proper orthogonal decomposition method. Journal of Fluids and Structures, $18,227-250$.

Antoulas, A.C., \& Sorensen, D.C. (2001). Approximation of large-scale dynamical systems: An overview. International Journal of Applied Mathematics and Computer Science, 11(5), 1093-1121.

Batoz, J.-L., \& Dhatt, G. (1992). Modeling of structures by the finite element method (Vol. 3: shells). Paris: Hermès.

Boumediene, F., Duigou, L., Boutyour, E.H., Miloudi, A., \& Cadou, J.M. (2011). Nonlinear forced vibration of damped plates coupling asymptotic numerical method and reduction models. Computational Mechanics, 47(4), 359-377.

Boumediene, F., Miloudi, A., Cadou, J.M., Duigou, L., \& Boutyour, E.H. (2009). Nonlinear forced vibration of damped plates by an asymptotic numerical method. Computers \& Structures, 87, 15081515.

Brezillon, A., Girault, G., \& Cadou, J.M. (2010). A numerical algorithm coupling a bifurcating indicator and a direct method for the computation of Hopf bifurcation points in fluid mechanics. Computers \& Fluids, 39(7), 1226-1240.

Cadou, J.M., Cochelin, B., Damil, N., \& Potier-Ferry, M. (2001). Asymptotic numerical method for stationary Navier-Stokes equations and with Petrov-Galerkin formulation. International Journal of Numerical Methods in Engineering, 50, 825-845.

Cadou, J.M., \& Potier-Ferry, M. (2010). A solver combining reduced basis and convergence acceleration with applications in non-linear elasticity. International Journal for Numerical Methods in Biomedical, 26, 1604-1617.

Cochelin, B., Damil, N., \& Potier-Ferry, M. (1994). Asymptotic numerical methods and Padé approximants for non-linear elastic structures. International Journal for Numerical methods in Engineering, 37, 1187-1213.

Cochelin, B., \& Vergez, C. (2009). A high order purely frequency-based harmonic balance formulation for continuation of periodic solutions. Journal of Sound and Vibration, 324, 243-262.

Daridon, L., Wattrisse, B., Chrysochoos, A., \& Potier-Ferry, M. (2011). Solving fracture problems using an asymptotic numerical method. Computers \& Structures, 89(5-6), 476-484.

Elhage-Hussein, A., Potier-Ferry, M., \& Damil, N. (2000). A numerical continuation method based on Padé approximants. International Journal of Solids and Structures, 37, 6981-7001.

Goncalves, P.B., Silva, F.M.A., \& Del Prado, Z.J.G.N. (2008). Low-dimensional models for the nonlinear vibration analysis of cylindrical shells based on a perturbation procedure and proper orthogonal decomposition. Journal of Sound and Vibration, 315, 641-663.

Holmes, P., Lumley, J.L., \& Berkooz, G. (1996). Turbulence, coherent structures, dynamical systems and symmetry. Cambridge: Cambridge University Press.

LaBryer, A., \& Attar, P.J. (2010). A harmonic balance approach for large-scale problems in nonlinear structural dynamics. Computers \& Structures, 88, 1002-1014.

Lall, S., Krysl, P., \& Marsden, J.E. (2003). Structure-preserving model reduction for mechanical systems. Physica D: Nonlinear Phenomena, 184, 304-318.

Médale, M., \& Cochelin, B. (2009). A parallel computer implementation of the asymptotic numerical method to study thermal convection instabilities. Journal of Computational Physics, 228(22), 82498262 .

Mickens R.E. (2010). Truly nonlinear oscillations. Harmonic Balance, parameter expansions, iteration, and averaging methods. USA: World Scientific Publishing Co. Pte. Ltd.

Mottaqui, H., Braikat, B., \& Damil, N. (2010). Discussion about parametrization in the asymptotic numerical method: Appmlication to nonlinear elastic shell. Computer Methods in Applied Mechanics and Engineering, 199(25-28), 1701-1709.

Potier-Ferry, M., Damil, N., Braikat, B., Descamps, J., Cadou, J.-M., Lei Cao, H., \& Elhage Hussein, A. (1997). Treatment of strong non-linearities by the asymptotic numerical method. Comptes Rendus de l'Académie des Sciences - Series II, b324(3), 171-177.

Wriggers, P. (2008). Nonlinear finite element methods. Berlin: Springer-Verlag.

Yvonnet, J., \& He, Q.C. (2007). The reduced model multiscale method (R3M) for the non-linear homogenization of hyperelastic media at finite strains. Journal of Computational Physics, 223, 341-368. 